\title{
Junge Psychoanalyse am PSZ
}

\section{Eine Generation von PsychoanalytikerInnen zwischen Identifikation und Frustration \\ Lutz Wittmann (Zürich), Nicole Burgermeister (Zürich) \\ und Monika Gsell (Zürich)}

Das Psychoanalytische Seminar Zürich (PSZ) gilt als Kind der 68-Bewegung. Es ist aus dem Protest gegen das psychoanalytische Establishment mit seinen verfestigten Strukturen hervorgegangen. Seither ist es zu einem festen Bestandteil der deutschsprachigen Psychoanalyse geworden, einige seiner ExponentInnen werden über die Sprachgrenze hinaus wahrgenommen. Wie aber gestaltet sich das Verhältnis der damals Aufgebrochenen zur nachkommenden Generation der jungen PsychoanalytikerInnen von heute? Wie nehmen TeilnehmerInnen, die noch nicht seit eh und je am PSZ ein- und ausgehen, ihr Seminar wahr?

Beim nachfolgenden Text handelt es sich um eine Ausarbeitung der Beiträge, mit welchen sich der im Wintersemester 2008/09 gegründete Treffpunkt «Junge Psychoanalyse» an der PSZ-Teilnehmerversammlung vom 30.01.2009 vorstellte.

\section{Ambivalenz: Identifikation und...}

Das Verhältnis von uns (angehenden) PsychoanalytikerInnen zum PSZ wollen wir mit dem Begriff der Ambivalenz beschreiben. So ist unser Engagement am PSZ und im Treffpunkt «Junge Psychoanalyse» zum einen geprägt von unserer Identifikation mit dieser Institution und der Dankbarkeit gegenüber denen, die sie aufgebaut und gestaltet haben. Wir haben hier so etwas wie unsere psychoanalytische Heimat gefunden. Wir schätzen den dialektischen Zugang zur Psychoanalyse sowie die Konfliktkultur, welche am PSZ gepflegt werden. Ebenso unterstützen wir die Vermeidung von hierarchischen Strukturen und die basisdemokratische Selbstverwaltung, welche uns das gleiche Stimmrecht einräumt wie denen, die von Anfang an dabei waren. Wir begrüssen, dass das PSZ nicht kommerziell ausgerichtet ist, auch deshalb, weil es als Aus- und Weiterbildungsstätte unschlagbar günstig ist. Ein weiterer Aspekt, den wir am PSZ geniessen, ist seine interdisziplinäre, praxisnahe Ausrichtung, die einem verschulten oder akademisierten Ansatz gegenüber steht. Und schliesslich sind wir allen AnalytikerInnen dankbar, die uns im Rahmen ihres ehrenamtlichen Engagements und zahlreicher spannender Kurse von ihrer 
Erfahrung und ihrem Wissen profitieren lassen. Diese Tradierung der Psychoanalyse stellt uns in einen professionellen und persönlichen Zusammenhang mit ihnen und der hier gepflegten kritischen Psychoanalyse.

\section{... Frustration}

Unsere Identifikation mit dem PSZ ist jedoch auch immer wieder mit Frustrationen verbunden. Zwar gilt unsere Stimme an Teilnehmerversammlungen bei Abstimmungen gleich viel wie jede andere. Aber häufig üben wir unser Stimmrecht gar nicht erst aus, da der Besuch von Teilnehmerversammlungen mit vielen Enttäuschungen verbunden ist: Kaum jemand stellt sich uns vor, nur wenige scheinen sich für unsere Anwesenheit zu interessieren, und unsere (seltenen) Voten werden unbeachtet übergangen. Wir spüren, es gibt hier unterschiedliche Gruppierungen, die ihre jeweiligen Interessen vertreten, aber mangels Aufklärung durchschauen wir die Verhältnisse nicht. (Eine der wenigen Gruppierungen, die wir auch als «Junge» unschwer erkennen, ist die Fraktion der «Silberrücken»: diese wie bei so vielen Gattungen männlich dominierte Gruppe ist an der Teilnehmerversammlung meist vorne links lokalisiert. Ihre Vertreter sind so wichtig für die Psychoanalyse, dass ihr Äusseres sich bereits dem unseres Urvaters annähert, und ihren Worten kommt so viel Gewicht zu, dass sie beim Reden aufstehen müssen, um sich diesem entgegenzustemmen.) Wir stellen fest, dass das Ideal der Vermeidung von Hierarchien oder Machtstrukturen leichter formuliert als erreicht ist. Mehr noch: da diese Grundaspekte menschlichen Sozialverhaltens am PSZ gar nicht vorgesehen sind, ist für uns Jüngere nur schwer durchschaubar, wer wo welche Fäden zieht. Wir merken, auch am PSZ gibt es Intrigen, etwa wenn eine offiziell eingesetzte Kommission umgangen wird, aber wir kriegen nicht wirklich mit, was läuft. Und immer wieder lässt man uns spüren, dass wir ja nur Anfänger sind. Dies leitet über zum Realitätsbezug, zum Bezug auf unsere Realität. Manchmal haben wir den Eindruck, dass unter den alten Hasen am PSZ ein «psychoanalytischer Autismus» grassiert. Da bleibt wenig Platz für die Anerkennung unserer heutigen Arbeitsbedingungen. Wir werden kaum in dem Einsatz unterstützt, mit welchem viele von uns sich in ihren Institutionen Freiräume für psychoanalytisches Denken und Arbeiten schaffen. (Als lobenswerte Ausnahme von dieser Feststellung begrüssen wir die Veranstaltung «Psychoanalyse in Institutionen.») Statt dessen spüren wir Geringschätzung, weil wir nicht schon vor Abschluss des Studiums eine psychoanalytische Praxis aufgebaut haben, bei der uns hochfrequente SelbstzahlerInnen die Türe einrennen. 
Wir sehen dankbar, was für grosse Anstrengungen das PSZ auf sich nimmt, um sich und unsere Ausbildung den Anforderungen unserer Realität anzupassen. Aber wenn sich dann unsere Realität und die Wahrnehmung derselben innerhalb des PSZ nicht decken, kommt es zu Enttäuschungen. Entsprechend schwierig gestaltet sich aus unserer Sicht der Aussenauftritt des PSZ. Manchmal möchte man meinen, das PSZ sei gar nicht an neuen TeilnehmerInnen interessiert - andernfalls würde es sich besser auf deren Situation einstellen. Abschliessend bleibt zu ergänzen, dass wir neben den vielen lehrreichen Kursen auch solche erlebt haben, in denen Umgangsformen oder didaktisches Konzept der DozentInnen Wünsche offen liessen. Es wirkt wenig einladend, wenn etwa DozentInnen nach langem Tuscheln zur Prüfung der Teilnahmevoraussetzungen übergehen, ohne vorher Zeit für eine Vorstellung ihrer selbst gefunden zu haben. Und wenn das eigene Nichtverstehen im Kreise der PublikumsnachbarInnen durch Achselzucken oder fragende Mimik vervollständigt wird, könnte durchaus die Frage aufkommen, ob hier ein grundsätzlicheres didaktisches Problem vorliegt.

\section{Wo sind die Jungen?}

Wenn an Teilnehmerversammlungen die «Alten» von den «Jungen» sprechen, diese aber weder an den Teilnehmerversammlungen, noch in den Ressortgruppen oder bei der Institutionsanalyse angetroffen werden, kommt die Frage auf: Wo sind die «Jungen»? Wie soll die Zukunft des PSZ aussehen, damit es nicht die eines Psychoanalytischen Seniorenheims Zürich wird? Und wer sind diese «Jungen»? Herrscht unter ihnen ein Konsumdenken, bei dem das Sammeln von Kurs-Testaten und Ausbildungsbestätigungen keinen Platz lässt für ein Engagement am PSZ? Oder lässt vielmehr das PSZ den «Jungen» keinen Platz? Entlässt die Revolution ihre Kinder in die Bedeutungslosigkeit?

Seit November 2008 findet an jedem ersten Montag eines Monats der Treffpunkt «Junge Psychoanalyse» am PSZ statt. An der ersten Sitzung kamen acht Leute, inzwischen sind es doppelt so viele, alle mit sehr unterschiedlichen Ausbildungen und beruflichen Hintergründen und unterschiedlich langer PSZErfahrung. Diese Leute sind am PSZ, weil sie sich - Weiterbildung hin oder her - intensiv mit Psychoanalyse als Theorie und Praxis auseinandersetzen, sich am PSZ vernetzen und integrieren und die Zukunft des PSZ mitgestalten wollen.

\section{Bedürfnisse und Anliegen}

Eines der dringendsten Bedürfnisse, so hat sich in diesen ersten Treffen gezeigt, ist der Austausch unter uns jüngeren Teilnehmerinnen und Teilnehmern 
zu uns beschäftigenden Themen und Anliegen. Dazu gehören Ausbildungsfragen ebenso wie die Auseinandersetzung mit unserer gegenwärtigen beruflichen Situation und inhaltlichen Aspekten psychoanalytischer Theorie und Praxis. Geplant sind zudem Informations- und Diskussionsabende zu spezifischen Themen wie etwa Anerkennungs- und Praxisbewilligungsverfahren oder zur Stellensuche. Darüber hinaus hoffen wir, mit dem Treffpunkt eine Anlaufstelle für neue Teilnehmerinnen und Teilnehmer anbieten und diese von Anfang an besser ins PSZ integrieren zu können, als es bisher der Fall ist.

Mit eigenen Intervisionsgruppen für junge und angehende AnalytikerInnen wollen wir zudem zusätzliche Räume schaffen, in denen der Austausch über die eigene Arbeit mit PatientInnen in einem gleichberechtigten und unterstützenden Rahmen stattfinden kann. Dass hieran Bedarf ist, zeigen die von uns gemachten sehr unterschiedlichen Erfahrungen bei der Teilnahme an klinischen Kursen und Intervisionsgruppen. So stellt es aus unserer Sicht eine besondere Stärke des PSZ dar, dass es hier Kurse und Gruppen gibt, in denen ein gleichberechtigter und fruchtbarer Austausch zwischen jüngeren und älteren TeilnehmerInnen stattfinden kann. Gleichzeitig vermissen manche von uns Orte am PSZ, welche die angstfreie Auseinandersetzung mit Fragen zur klinischen Praxis, die jüngere TeilnehmerInnen so dringend benötigten, ermöglichen.

\section{Interesse wecken}

Es ist uns ein besonderes Anliegen, jüngere PsychologInnen, MedizinerInnen und besonders auch VertreterInnen anderer Berufsgruppen (WissenschaftlerInnen, SozialarbeiterInnen, PädagogInnen, etc.) weiterhin für die Psychoanalyse und das PSZ zu interessieren. Der Treffpunkt will ein Ort sein, an dem sich die VertreterInnen all dieser verschiedenen Berufsgruppen über die je unterschiedlichen Herausforderungen, Psychoanalyse zu praktizieren, austauschen können. Dabei soll ein Wissen um und eine Akzeptanz für diese unterschiedlichen Situationen geschaffen werden - etwas, was wir sonst am PSZ vermissen. Es ist unser Anliegen, dass der Graben, der sich zwischen den VertreterInnen der PSYBerufe und denen anderer Berufsgruppen aufzutun droht, gar nicht erst entsteht oder rechtzeitig wieder überbrückt werden kann.

Auch wenn der Psychoanalyse angesichts der gegenwärtigen Entwicklungen im Gesundheitswesen, zunehmend restriktiveren Psychotherapiegesetzgebungen und einer breiten Konkurrenz an als effizienter geltenden Therapierichtungen ein härtererWind entgegenschlägt als noch vor zwanzig, dreissig Jahren, sind wir zuversichtlich, dass die Psychoanalyse mit ihrer spezifischen Zugangsweise zu Menschen 
und menschlichem Leid auch weiterhin ihren Platz in der Gesellschaft haben wird. Ebenso sind wir der Überzeugung, dass die von uns als Stärken wahrgenommenen Besonderheiten der Ausbildung am PSZ angesichts der steigenden Tendenz zu verschulten Ausbildungsgängen auch weiterhin über Anziehungskraft verfügen. Ein aktives und selbstbewusstes Zugehen auf Studierende scheint uns hier jedoch dringend notwendig. Aus diesem Grund setzen wir uns ein für eine stärkere Präsenz einer «jungen Psychoanalyse» an Universitäten und Fachhochschulen und entwerfen derzeit gemeinsam mit erfahreneren PSZ-TeilnehmerInnen spezielle Angebote für Studierende. Geplant sind etwa die Einführung von Schnuppersemestern, die Möglichkeit zur Teilnahme an Intervisionsgruppen sowie Einführungskurse, in denen Studierenden ein praxisorientierter Einblick in die tägliche Arbeit von PsychoanalytikerInnen ermöglicht werden soll.

\section{Weiterbildung und Ausbildung}

Damit das PSZ auch weiterhin attraktiv bleibt für Leute, die nicht aus den PSY-Berufen kommen, scheint es uns wichtig, der Ausbildung als einem seit je zentralen und identitätsstiftenden Angebot des PSZ wieder mehr Gewicht zu geben. Zur Zeit ist es so, dass zwar immer noch dauernd von «Ausbildung» die Rede ist, faktisch aber «Weiterbildung» gemeint ist. (Ein Beispiel hierfür ist die PSZ-Homepage, wo es zwar einen Link «Ausbildungen» gibt, auf der betreffenden Site selbst aber ausschliesslich von der Weiterbildung die Rede ist, inklusive einem Download-Dokument «Weiterbildungskonzept», aber weit und breit kein «Ausbildungskonzept».) Wir verstehen, dass die Frage der Weiterbildung viel Energie absorbiert hat und für das Selbstverständnis des PSZ eine existentielle Herausforderung bedeutete. Wir denken aber auch, dass jetzt - wo das Weiterbildungskonzept steht und (soviel wir sehen) PSZ-intern breit akzeptiert ist - der Zeitpunkt gekommen ist, um das Verhältnis von Ausbildung und Weiterbildung zu reflektieren und darüber nachzudenken, in welcher Form das implizite Ausbildungskonzept expliziert und sichtbar gemacht werden kann und ob es auch einiger Neuerungen bedarf, um den veränderten gesellschaftlichen Bedingungen gerecht zu werden. Diese Auseinandersetzung, die wir gerne zusammen mit den «Älteren» am PSZ führen möchten, wäre auch eine Gelegenheit für diese, die Geschichte des PSZ - in der das spezifische Ausbildungskonzept des Seminars ja eine zentrale Rolle spielt - an die Jungen weiter zu geben.

Der Treffpunkt «Junge Psychoanalyse» will also den Austausch unter den jüngeren und neueren TeilnehmerInnen am PSZ sowie die Entwicklung von eigenen Positionen fördern. Er will aber auch in den Dialog mit den «Alten», den etablierten 
TeilnehmerInnen treten:Wir wollen unsere Anliegen einbringen, wollen gehört und gesehen werden, andererseits aber auch sichtbare AnsprechpartnerInnen sein für die Anliegen der «Alten» gegenüber den «Jungen».

Der Treffpunkt «Junge Psychoanalyse» findet an jedem ersten Montag des Monats (auch in den Semesterferien) 20:30-22:00h statt. 